\title{
Predictive biomarkers of response for PD-1/PD-L1 inhibitors: a cumbersome gold rush
}

\author{
Andrea Vingiani ${ }^{1}$, Massimo Barberis ${ }^{1}$, Elena Guerini-Rocco ${ }^{1,2}$
}

\begin{abstract}
Programmed cell death protein 1 (PD-1) and its ligand programmed cell death-ligand 1 (PD-L1) are overexpressed in a number of human malignancies. More interestingly, their expression has been associated with patient survival in non-small cell lung cancer (NSCLC), melanoma, renal cell carcinoma, esophageal, pancreatic and colorectal carcinoma, with the data commonly suggesting a negative prognostic role. In this review, we summarize the pros and cons regarding the predictive role of PD-L1 expression in candidate patients for checkpoint inhibitors. Furthermore, we discuss the potential predictive role of other biomarkers, such as tumor mutational burden, microsatellite instability, mismatch repair deficiency and tumor infiltrating lymphocytes. We conclude that PD-L1 testing probably represents simply a "snapshot" of an intricate, fluctuating and dynamic process, that in turn represents the interplay between the immune system and cancer. The PD-L1 assay can be considered more useful for response stratification than in patient selection.
\end{abstract}

Key words: biomarkers, immunotherapy, PD-L1

\section{Introduction}

Programmed cell death protein 1 (PD-1 or CD279) and its ligand programmed cell death-ligand 1 (PD-L1 or CD274 or B7-H1) are cell surface transmembrane molecules playing a pivotal role in down-regulating the immune system, thus promoting immune tolerance by suppressing $\mathrm{T}$ cell inflammatory activity and preventing autoimmunity in physiological circumstances [1-3].

Activation of the PD1 - PD-L1 axis results in increased apoptosis of $\mathrm{T}$ lymphocytes, reduced apoptosis in regulatory $\mathrm{T}$ cells (Tregs), reduced $\mathrm{T}$ cell proliferation and interferon (IFN)- $\gamma$ secretion $[1,4-6]$.

The expression of PD1 and PDL-1 on cancer cells and on immune cells in the tumor microenvironment is assumed to represent a crucial player of the negative feedback loop ultimately leading to the so-called "immune escape", and re-

'Department of Pathology, European Institute of Oncology, Milan, Italy.

2University of Milan, Department of Oncology and Hemato-oncology, Milan, Italy.

Correspondence to:

Massimo Barberis,

Istituto Europeo di Oncologia,

Via Ripamonti 451, 20141 Milano, Italy.

Phone: +3902 57489414

E-mail: massimo.barberis@ieo.it

CANCER BREAKING NEWS 2017;5(3):14-19

DOI: 10.19156/cbn.2017.0054 sulting in uncontrolled cancer growth and progression [7-9]. PD1 and PD-L1 are overexpressed in a number of human malignancies; more interestingly, their expression has been associated with patient survival in non-small cell lung cancer (NSCLC), melanoma, renal cell carcinoma, esophageal, pancreatic and colorectal carcinoma, with the data commonly suggesting a negative prognostic role [10-17].

In this scenario, a number of PD1 inhibitors, namely nivolumab and pembrolizumab, and PD-L1 inhibitors, such as atezolizumab and avelumab, have demonstrated promising therapeutic activity and are currently available for the treatment of several advanced-stage neoplasms, such as NSCLC, melanoma, urothelial carcinoma, renal cell carcinoma, head and neck cancer and Hodgkin lymphoma [18-28].

Despite the unprecedented survival benefit obtained with checkpoint inhibition, nearly $40-60 \%$ of patients will not gain advantage from these therapies, that are furthermore costly and not free from toxicities. In this context, reliable and cost-effective predictive biomarkers are therefore highly desirable, and the assessment of PD-L1 expression by immunohistochemistry (IHC) seems to represent the most widely used and validated biomarker thus far.

\section{PD-1/PD-L1 expression by ICH and the NSCLC paradigm}

A number of different IHC assays have been developed to assess the expression of PD-1 and PD-L1, and different 
Table 1. Immunohistochemistry assays for assessing the expression of PD-1 and PD-L1.

\begin{tabular}{lccccc}
\hline Manufacturer & $\begin{array}{c}\text { Anti-PD-1/ } \\
\text { PD-L1 Drug }\end{array}$ & $\begin{array}{c}\text { IVD } \\
\text { Partner }\end{array}$ & Ab Clone & Cutoffs & Cell Scored \\
\hline AstraZeneca & Durvalumab & Ventana & SP263 & $25 \%$ & Tumor cells \\
\hline Bristol Myers Squibb & Nivolumab & Dako & $28-8$ & $1 \%, 5 \%, 10 \%$ & Tumor cells \\
\hline Merck & Pembrolizumab & Dako & $22 \mathrm{C} 3$ & $1 \%, 50 \%$ & Tumor cells \\
\hline Merck KGaA and Pfizer & Avelumab & Dako & - & - & Tumor cells and TILs \\
\hline Roche & Atezolizumab & Ventana & SP142 & $50 \%$ of tumor cells, 5\% in TILs & Tumor cells and TILs \\
\hline
\end{tabular}

Ab: antibody; IVD: in vitro diagnostic; PD-1: programmed cell death protein 1; PD-L1: programmed cell death-ligand 1; TILs: tumor-infiltrating lymphocytes.

clones, staining protocols, platforms, scoring systems, and thresholds have been introduced for and linked to specific inhibitors (Table 1). In patients with NSCLC, data stemming from clinical trials and large pooled analyses have shown a positive correlation between PD-L1 expression and the clinical benefit deriving from PD-1/PD-L1 inhibition [29-33].

The PD-L1 IHC 22C3 PharmDx assay has been approved by the US Food and Drug Administration (FDA) as the exclusive companion diagnostic for pembrolizumab (Figure 1).

In the KEYNOTE 024 trial, 305 patients with untreated advanced NSCLC, with PD-L1 expression on at least 50\% of tumor cells were randomized to receive either pembrolizumab or platinum-based chemotherapy. Investigators showed that pembrolizumab was associated with significantly longer progression-free (hazard ratio [HR] 0.50; $95 \%$ confidence interval $[\mathrm{CI}] 0.37-0.68 ; \mathrm{p}<0.001)$ and overall survival (HR $0.60 ; 95 \%$ CI $0.41-0.89 ; \mathrm{p}=0.005$ ) and

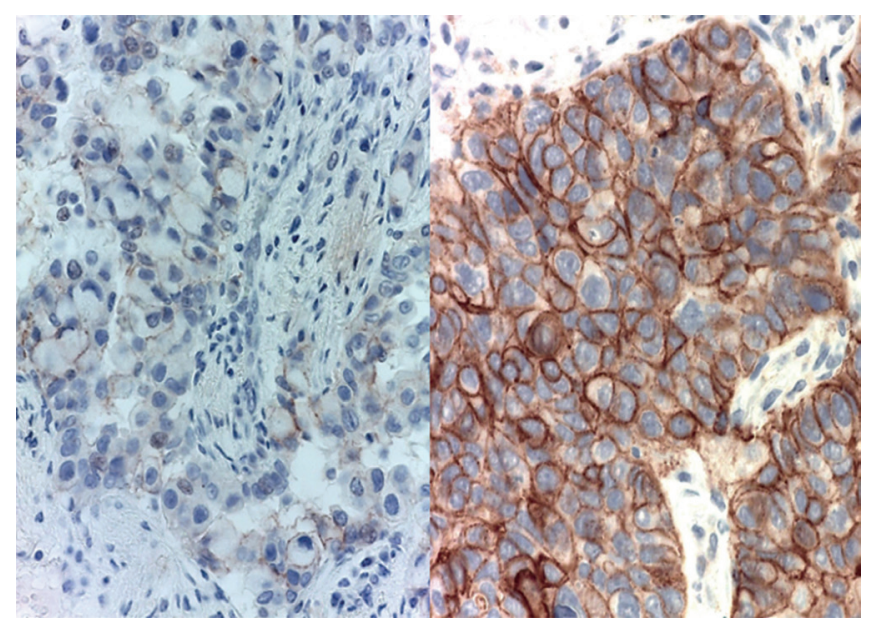

Fig. 1. Two typical examples of immunohistochemical staining with the programmed death ligand 1 immunohistochemical 22C3 PharmDx test (Agilent) on the Dako Autostainer, showing on the left a case with weak and incomplete cell membrane staining, and on the right an intense and complete staining. with fewer adverse events than with platinum-based chemotherapy [34].

Similarly, PD-L1 IHC 28-8 PharmDx (Agilent Technologies, Santa Clara, CA/Dako Carpinteria, CA) has been approved as the companion test for nivolumab, given the clinical evidence of patients affected by NSCLC showing positivity for PD-L1 in 1\% or more tumor cells. Notably, data coming from pivotal clinical trials showed that a significant subset of PD-L1-negative patients clearly gained an advantage from treatment with nivolumab [35-37]. Consequently, the FDA label for nivolumab did not specify any cut-off for PD-L1 positivity. The phase III Checkmate 026 study similarly compared the anti-PD-1 antibody nivolumab as a single agent with platinum-based chemotherapy in firstline PD-L1 $>5 \%$ positive NSCLC. The primary endpoint of progression-free survival (PFS) in the population with PD$\mathrm{L} 1>5 \%$ was not met (HR 1.15, $\mathrm{p}=0.25$ ) [38].

The complementary diagnostic Ventana PD-L1 SP142 antibody (Ventana Medical Systems, Tucson, AZ) has been approved for therapy with atezolizumab, in consideration of the enhanced survival observed in patients with NSCLC presenting at least $50 \%$ of tumor cells expressing PD-L1 or at least $10 \%$ of the tumor area occupied by PD-L1-expressing tumor-infiltrating immune cells. Although these assays may provide useful information to clinicians as complementary diagnostics, neither the PD-L1 IHC 28-8 PharmDx nor the Ventana PD-L1 (SP142) assay are strictly required for treatment with nivolumab or atezolizumab, respectively. Finally, the Ventana SP263 clone is still under the FDA regulatory process and has been developed for treatment with durvalumab by using a positivity cutoff of $25 \%$ or more tumor cells [31].

From a practical point of view, what emerges is the number and heterogeneity of the antibody clones and platforms used, thereby giving rise to concerns about the reproducibility and robustness of different assays evaluating the expression of the same molecule and aimed at predicting clinical response to therapies sharing the same mechanism 
of action. For example, according to its FDA label, pembrolizumab administration is linked to an approved IHC test with the $22 \mathrm{C} 3$ antibody, run on the DAKO platform, that in turn not all laboratories around the world are provided with. The "Blueprint PD-L1 IHC Assay Comparison Project" compared the performances of 4 assays, namely SP263, 28-8, SP142 and 22C3, and revealed that three of the four assays were closely aligned on tumor cell staining whereas the fourth showed lower sensibility [39]. A number of harmonization studies followed, suggesting that some of IHC assays could be interchangeable, but these data still lack clinical validation [40-43]. Interestingly, Marchetti and colleagues found a high correlation between PD-L1 expression data obtained with the Agilent PD-L1 IHC 22C3 PharmDx and the Ventana PD-L1 (SP263) tests in NSCLC, thus suggesting that the two assays could potentially be utilized interchangeably.

Another drawback of note is the vast heterogeneity of the threshold used. To date, there is no consensus about the relevance of patterns of expression (on cancer cells, membranous or cytoplasmic, and immune cells) and different quantitative cutoffs have been variably used and approved by regulatory authorities. The Blueprint study highlighted that, despite similar analytical performance of PD-L1 expression for three assays, interchanging assays and cutoffs could lead to "misclassification" of PD-L1 status for a not-negligible subset of patients. Moreover, data stemming from clinical trials showed how some thresholds used could lead to the risk of exclusion of a considerable number of responders. In this context, it may make more sense to consider PD-L1 expression as a continuous variable rather than a "on" or "off" indicator. Probably this assay can be considered more useful for response stratification than in patient selection.

\section{PD-1/PD-L1 testing in other malignancies}

Checkpoint inhibition is one of the currently approved standards of care for advanced stage patients with malignant melanoma (MM). To date, no validated predictive biomarker of response exists $[44,45]$. Nevertheless, the absence of PD-L1 expression on malignant and immune cells has been shown to predict a poor, non-existent response to PD-1 inhibition [46]. In their meta-analysis, Gandini et al. showed that objective response rates in patients with MM were significantly higher in PD-L1-positive than in PD-L1-negative tumors (45\% vs 27\%, respectively) [47]. Furthermore, recent evidence suggests that low PD-L1 expressing MM patients could derive greater benefit from dual checkpoint inhibition with nivolumab plus ipilimumab [48, 49].

Nivolumab has been registered for the treatment of advanced renal cell carcinoma (RCC); in this setting, the expression of PD-L1 was prognostic but still not predic- tive of clinical benefit deriving from checkpoint inhibition $[50,51]$.

With regard to urothelial carcinoma, the predictive value of PD-L1 expression varied across different studies. Rosenberg et al. showed that the overexpression of PD-L1 was predictive of clinical benefit in a second-line trial randomizing patient to receive atezolizumab [52], while in the firstline setting, no differences in efficacy according to PD-L1 expression were noted [53]. Several other checkpoint inhibitors have been validated in urothelial cancer, and again, evidence on the putative predictive value of PD-L1 expression is far from exhaustive.

\section{Alternative biomarker assays}

Recent evidence suggests that mutational burden could be positively associated with response to checkpoint inhibitors. In patients with NSCLC, Rizvi et al. demonstrated that tumors with high numbers of non-synonymous mutations showed better PFS and objective response rate in contrast to tumors with low mutational burden $[54,55]$. Similarly, copy number variations, expressed as a quantitative chromosomal number instability (CNI) in tumor cell-free DNA obtained from liquid biopsy have been shown to predict benefit from immunotherapy [56].

Mismatch repair deficiency (MMRD)-driven tumors are associated with high mutational load, and therefore with overt immunogenicity due to a large amount of "neo-epitopes". In this regard, Le et al. found MMRD to be predictive of response to pembrolizumab in patients with colorectal cancer [57]. These findings, along with data from 149 patients enrolled in five clinical trials, led to the FDA approval of pembrolizumab for any MMR-deficient solid cancer, thus perhaps representing the first example in the history of oncology of a therapeutic indication relying on a biological biomarker rather than the location of the neoplasm [58].

Tumor-infiltrating lymphocytes (TILs) have been positively correlated with prognosis and treatment response, especially in the neoadjuvant setting, in a large number of neoplasms. Interestingly, in a pivotal study by Tumeh et al., it was demonstrated in MM that the prevalence of CD8+ $\mathrm{T}$ cytotoxic lymphocyte was predictive of anti PD-1 therapy benefit, outperforming the assessment of PD-1 expression [59], thus suggesting a possible role for TILs as a biomarker in PD-1/PD-L1 inhibition. From a speculative point of view, the presence of immune cells in the tumor microenvironment could mirror the immunogenicity of the neoplasm itself, being able therefore to pinpoint tumors amenable to anti-tumor immune response restoration through checkpoint inhibition. The identification of the lymphocytic subpopulation and the evaluation of the actual state of the activation/exhaustion of 
the immune system could provide valuable information. Two independent groups have demonstrated that $\mathrm{T}$ cell exhaustion is characterized by a specific genetic landscape, consisting of peculiar patterns of accessibility of gene regulatory elements to transcriptional factors [60-62]. These data led to the hypothesis that the existence of a window between an "initial" and reversible state of $\mathrm{T}$ cell exhaustion and a "permanent" state, during which patients could potentially take advantage of immune reactivation by anti-PD-1 drugs. Along this line, in the era of transcriptomics and of gene expression profiling, the assessment of PD-1/PD-L1 expression and of other markers of T cell activation and/or in tumor-associated T lymphocytes and/ or tumor cells (such as CTLA 4, TIM3, LAG3, TIGIT and so on) by more and more comprehensive "immune signatures" might be of great value [63]. For example, Ribas et al. described an interferon-inflammatory immune gene expression signature associated with both enhanced overall response rates and PFS in advanced MM patients receiving pembrolizumab [64].

Assessing immune-related blood parameters has shown some interesting results, especially in MM. Ferrucci et al. demonstrated that high absolute neutrophil count and derived neutrophil to lymphocyte ratio were significantly related to poorer overall survival and PFS in 720 patients with advanced MM treated with ipilimumab [65], while other groups showed that absolute lymphocyte count was associated with a better outcome [66, 67]. Similarly, high serum lactate dehydrogenase levels have been demonstrated to be a reliable predictor of poor patient outcomes in response to CTLA 4 and/or PD-1 blockade in patients with MM [68].

Encouraging data surrounds the putative predictive role of the identification of tumor antigen-specific antibodies, antigen-reactive T-cells [69], the assessment of circulating lymphocyte subtypes [70] and the microbiome profile [71].

\section{References}

1. Freeman GJ, Long AJ, Iwai Y et al. Engagement of the PD-1 immunoinhibitory receptor by a novel B7 family member leads to negative regulation of lymphocyte activation. J Exp Med 2000;192:1027-34.

2. Keir ME, Freeman GJ, Sharpe A. PD-1 regulates self-reactive CD8+ $\mathrm{T}$ cell responses to antigen in lymph nodes and tissues. J Immunol 2007;179:5064-70.

3. Sharpe AH, Wherry EJ, Ahmed R, Freeman GJ. The function of programmed cell death 1 and its ligands in regulating autoimmunity and infection. Nat Immunol 2007;8:239-45

4. Francisco LM, Sage PT, Sharpe AH. The PD-1 pathway

\section{Conclusions}

Checkpoint inhibitors have entered forcefully into the clinical arena for the treatment of several human malignancies, showing notable results. Still, only a fraction of the treated population gain advantage from these expensive therapies, which are not without side effects. The identification of reliable biomarkers of response is therefore crucial for selecting the target population. To date, PD-1/PD-L1 testing by IHC represents the most widely used biomarker, although it suffers from obvious limitations. PD-L1 testing is mainly performed on small biopsy samples, sometimes on fine needle biopsy, with the risk of not being representative of the entire tumor or of the numerous metastatic localizations. As well as being inadequate in deciphering the topographical heterogeneity of human malignancies, PD-L1 testing represents simply a "snapshot" of an intricate, fluctuating and dynamic process that in turn represents the interplay between the immune system and cancer. The status of the PD-1/PD-L1 axis can, furthermore, be influenced by several factors, namely disease stage, previous lines of therapy, and any eventual concomitant therapy such as immunosuppressive drugs. In this scenario, it is, therefore, reasonable to apply our efforts in seeking for biomarkers capable of pinpointing tumor actually amenable to immune activity restoration, taking into consideration the biology of the tumor cells themselves, the status of the immune system, and their protean interaction.

\section{Acknowledgments}

The Authors thank Ray Hill, an independent medical writer, who provided native English editing and journal styling on behalf of HPS.

\section{Conflicts of Interest}

The Authors declare there are no conflicts of interest in relation to this article.

in tolerance and autoimmunity. Immunological Reviews 2010;236:219-42.

5. Carter L, Fouser LA, Jussif J et al. PD-1:PD-L inhibitory pathway affects both $\mathrm{CD} 4(+)$ and $\mathrm{CD} 8(+) \mathrm{T}$ cells and is overcome by IL-2. Eur J Immunol 2002:32(3):634-43.

6. Dong H, Strome SE, Salomao DR et al. Tumor-associated B7-H1 promotes T-cell apoptosis: a potential mechanism of immune evasion. Nature Med 2002;8(8):793-800.

7. Schreiber RD, Old LJ, Smyth MJ. Cancer immunoediting: integrating immunity's roles in cancer suppression and promotion. Science 2011;331(6024):1565-70.

8. Dunn GP, Bruce AT, Ikeda H. Cancer immunoediting: 
from immunosurveillance to tumor escape. Nat Immunol 2002;3:991-8.

9. Mittal D, Gubin MM, Schreiber RD, Smyth MJ. New insights into cancer immunoediting and its three component phaseselimination, equilibrium and escape. Current Opin Immunol 2014;27:16-25.

10. Thompson RH, Dong H, Lohse CM et al. PD-1 is expressed by tumor-infiltrating immune cells and is associated with poor outcome for patients with renal cell carcinoma. Clin Cancer Res 2007;13(6):1757-61.

11. Wu P, Wu D, Li L et al. PD-L1 and survival in solid tumors: A meta-analysis. PLoS One 2015 Jun 26;10(6):e0131403.

12. Wang A, Wang HY, Liu Y et al. The prognostic value of PDL1 expression for non-small cell lung cancer patients: a metaanalysis. Eur J Surg Oncol 2015;41(4):450-6.

13. Ohigashi Y, Sho M, Yamada Y et al. Clinical significance of programmed death-1 ligand-1 and programmed death-1 ligand-2 expression in human esophageal cancer. Clin Cancer Res 2005;11(8):2947-53.

14. Hino R, Kabashima K, Kato Y et al. Tumor cell expression of programmed cell death-1 ligand 1 is a prognostic factor for malignant melanoma. Cancer 2010;116(7):1757-66.

15. Shi SJ, Wang LJ, Wang GD et al. B7-H1 expression is associated with poor prognosis in colorectal carcinoma and regulates the proliferation and invasion of HCT116 colorectal cancer cells. PloS One 2013;8(10):e76012.

16. Zhou ZJ, Zhan P, Song Y et al. PD-L1 over-expression and survival in patients with non-small cell lung cancer: a metaanalysis. Transl Lung Cancer Res 2015;4(2):203-8.

17. Pan ZK, Ye F, Wu X et al. Clinicopathological and prognostic significance of programmed cell death ligand1 (PD-L1) expression in patients with non-small cell lung cancer: a metaanalysis. J Thorac Dis 2015;7(3):462-70.

18. Rittmeyer A, Barlesi F, Waterkamp D et al. Atezolizumab versus docetaxel in patients with previously treated non-smallcell lung cancer (OAK): a phase 3, open-label, multicentre randomised controlled trial. Lancet 2017;389:255-65.

19. Callahan MK, Postow MA, Wolchok JD. CTLA-4 and PD-1 pathway blockade: combinations in the clinic. Front Oncol 2014;4:385.

20. Wolchok JD, Kluger $\mathrm{H}$, Callahan $\mathrm{MK}$ et al. Nivolumab plus ipilimumab in advanced melanoma. $\mathrm{N}$ Engl $\mathrm{J}$ Med 2013;369:122-33.

21. Ferris RL, Blumenschein G Jr, Fayette J et al. Nivolumab for recurrent squamous-cell carcinoma of the head and neck. N Engl J Med 2016;375(19):1856-67.

22. Borghaei H, Paz-Ares L, Horn L et al. Nivolumab versus docetaxel in advanced nonsquamous non-small-cell lung cancer. N Engl J Med 2015;373(17):1627-39.

23. Sharma P, Retz M, Siefker-Radtke A et al. Nivolumab in metastatic urothelial carcinoma after platinum therapy (CheckMate 275): a multicentre, single-arm, phase 2 trial. Lancet Oncol 2017;18(3):312-22.

24. Reck M, Rodriguez-Abreu D, Robinson AG et al. Pembrolizumab versus chemotherapy for PD-L1-positive non-smallcell lung cancer. N Engl J Med 2016;375(19):1823-33.

25. Balar AV, Galsky MD, Rosenberg JE et al. IMvigor210 Study Group. Atezolizumab as first-line treatment in cisplatin-ineligible patients with locally advanced and metastatic urothelial carcinoma: a single-arm, multicentre, phase 2 trial. Lancet 2017;389(10064):67-76.

26. Apolo AB, Infante JR, Balmanoukian A et al. Avelumab, an antiprogrammed death-ligand 1 antibody, in patients with refractory metastatic urothelial carcinoma: results from a multicenter, Phase Ib Study. J Clin Oncol 2017;35(19):2117-24.

27. Bellmunt J, de Wit R, Vaughn DJ et al. KEYNOTE-045 Investigators. Pembrolizumab as second-line therapy for advanced urothelial carcinoma. N Engl J Med 2017;376(11):1015-26.

28. Chen R, Zinzani PL, Fanale MA et al. Phase II study of the efficacy and safety of pembrolizumab for relapsed/refractory classic Hodgkin lymphoma. J Clin Oncol 2017; 35(19):2125-32.

29. Grigg C, Rizvi NA. PD-L1 biomarker testing for non-small cell lung cancer: truth or fiction? J Immunother Cancer 2016;4:48.

30. Sacher AG, Gandhi L. Biomarkers for the clinical use of PD-1/PD-L1 inhibitors in non-small-cell lung cancer: a review. JAMA Oncol 2016;2:1217-22.

31. Patel SP, Kurzrock R. PD-L1 expression as a predictive biomarker in cancer immunotherapy. Mol Cancer Ther 2015;14:847-56.

32. Carbognin L, Pilotto S, Milella M et al. Differential activity of nivolumab, pembrolizumab and MPDL3280A according to the tumor expression of programmed death-ligand-1 (PD-L1): sensitivity analysis of trials in melanoma, lung and genitourinary cancers. PLoS One 2015;10:e0130142.

33. Abdel-Rahman O. Correlation between PD-L1 expression and outcome of NSCLC patients treated with anti-PD-1/ PD-L1 agents: a meta-analysis. Crit Rev Oncol Hematol 2016;101:75-85.

34. Reck M, Rodríguez-Abreu D, Robinson AG et al. Pembrolizumab versus chemotherapy for PD-L1-positive non-smallcell lung cancer. N Engl J Med 2016;375:1823-33.

35. Topalian SL, Hodi FS, Brahmer JR et al. Safety, activity, and immune correlates of anti-PD-1 antibody in cancer. N Engl J Med 2012;366:2443-54.

36. Borghaei H, Paz-Ares L, Horn L et al. Nivolumab versus docetaxel in advanced nonsquamous non-small-cell lung cancer. N Engl J Med 2015;373:1627-39.

37. Gettinger SN, Horn L, Gandhi L et al. Overall survival and long-term safety of nivolumab (anti-programmed death 1 antibody, BMS-936558, ONO-4538) in patients with previously treated advanced non-small-cell lung cancer. J Clin Oncol 2015;33:2004-12.

38. Carbone DP, Reck M, Paz-Ares L et al. First-line nivolumab in stage IV or recurrent non-small-cell lung cancer. N Engl J Med 2017;376:2415-26.

39. Hirsch FR, McElhinny A, Stanforth D et al. PD-L1 immunohistochemistry assays for lung cancer: results from phase 1 of the Blueprint PD-L1 IHC Assay Comparison Project. J Thorac Oncol 2017 Feb;12(2):208-22.

40. Scheel AH, Dietel M, Heukamp LC et al. Harmonized PDL1 immunohistochemistry for pulmonary squamous-cell and adenocarcinomas. Mod Pathol 2016;29:1165-72.

41. Ratcliffe MJ, Sharpe A, Midha A et al. Agreement between programmed cell death ligand-1 diagnostic assays across multiple protein expression cut-offs in non-small cell lung cancer. Clin Cancer Res 2017;23:3585-91. 
42. Adam J, Rouquette I, Damotte D et al. Multicentric French harmonization study for PD-L1 IHC testing in NSCLC [Abstract PLO4a]. J Thorac Oncol 2016;12:S11-S12.

43. Marchetti A, Barberis M, Franco R et al. Multicenter comparison of 22C3 PharmDx (Agilent) and SP263 (Ventana) assays to test PD-L1 expression for NSCLC patients to be treated with immune checkpoint inhibitors. J Thorac Oncol 2017;12(11):1654-63.

44. Swaika A, Hammond WA, Joseph RW. Current state of antiPD-L1 and anti-PD-1 agents in cancer therapy. Mol Immunol 2015;67(2 Pt A):4-17.

45. Kee D, McArthur G. Immunotherapy of melanoma. Eur J Surg Oncol 2017;43:594-603.

46. Sharma P, Hu-Lieskovan S, Wargo JA et al. Primary, adaptive, and acquired resistance to cancer immunotherapy. Cell 2017;168(4):707-23.

47. Gandini S, Massi D, Mandalà M. PD-L1 expression in cancer patients receiving anti PD-1/PD-L1 antibodies: A systematic review and meta-analysis. Crit Rev Oncol Hematol 2016;100:88-98.

48. Wolchok JD, Chiarion-Sileni V, Gonzalez R et al. Overall survival with combined nivolumab and ipilimumab in advanced melanoma. N Engl J Med 2017;377(14): 1345-56.

49. Ribas A, Martin-Algarra S, Bhatia S et al. Immunomodulatory effects of nivolumab and ipilimumab in combination or nivolumab monotherapy in advanced melanoma patients: CheckMate 038 [Abstract]. Presented at the American Association for Cancer Research Annual Meeting, Washington, DC, April 1-5, 2017.

50. Xu F, Xu L, Wang Q et al. Clinicopathological and prognostic value of programmed death ligand-1 (PD-L1) in renal cell carcinoma: a meta-analysis. Int J Clin Exp Med 2015; 8:14595-603.

51. Motzer RJ, Escudier B, McDermott DF et al. Nivolumab versus everolimus in advanced renal-cell carcinoma. N Engl J Med 2015;373:1803-13.

52. Rosenberg JE, Hoffman-Censits J, Powles T et al. Atezolizumab in patients with locally advanced and metastatic urothelial carcinoma who have progressed following treatment with platinum-based chemotherapy: a single-arm, multicentre, phase 2 trial. Lancet 2016;387:1909-20.

53. Balar AV, Galsky MD, Loriot Y. Atezolizumab (atezo) as first-line (1L) therapy in cisplatin-ineligible locally advanced/metastatic urothelial carcinoma (mUC): Primary analysis of IMvigor210 cohort 1 (Abstract). J Clin Oncol 2016;34(suppl):LBA4500.

54. Rizvi NA, Hellmann MD, Snyder A et al. Cancer immunology. Mutational landscape determines sensitivity to PD-1 blockade in non-small cell lung cancer. Science 2015;348(6230):124-8.

55. McGranahan N, Furness AJ, Rosenthal R et al. Clonal neoantigens elicit $\mathrm{T}$ cell immunoreactivity and sensitivity to immune checkpoint blockade. Science 2016;351(6280):1463-9.
56. Weiss GJ, Beck J, Braun DP et al. Tumor cell-free DNA copy number instability predicts therapeutic response to immunotherapy. Clin Cancer Res 2017;23(17):5074-81.

57. Le DT, Uram JN, Wang $\mathrm{H}$ et al. PD-1 blockade in tumors with mismatch-repair deficiency. N Engl J Med 2015;372(26):2509-20.

58. Lemery S, Keegan P, Pazdur R. First FDA approval agnostic of cancer site - when a biomarker defines the indication. N Engl J Med 2017;377(15):1409-12.

59. Tumeh PC, Harview CL, Yearley JH et al. PD-1 blockade induces responses by inhibiting adaptive immune resistance. Nature 2014;515:568-71.

60. Sen DR, Kaminski J, Barnitz RA et al. The epigenetic landscape of T cell exhaustion. Science 2016;354(6316):1165-9.

61. Pauken KE, Sammons MA, Odorizzi PM et al. Epigenetic stability of exhausted $\mathrm{T}$ cells limits durability of reinvigoration by PD-1 blockade. Science 2016;354(6316):1160-5.

62. Turner SJ, Russ BE. Can T cells be too exhausted to fight back? Science 2016;354(6316):1104-5.

63. Fridman WH, Zitvogel L, Sautès-Fridman C, Kroemer G. The immune contexture in cancer prognosis and treatment. Nat Rev Clin Oncol 2017;14(12):717-34.

64. Ribas A, Robert C, Hodi FS et al. Association of response to programmed death receptor 1 (PD-1) blockade with pembrolizumab (MK-3475) with an interferon-inflammatory immune gene signature [Abstract]. J Clin Oncol 2015;33(15 suppl):3001.

65. Ferrucci PF, Ascierto PA, Pigozzo J et al. Baseline neutrophils and derived neutrophil-to-lymphocyte ratio: prognostic relevance in metastatic melanoma patients receiving ipilimumab. Ann Oncol 2016;27(4):732-8.

66. Alexander M, Mellor JD, McArthur G, Kee D. Ipilimumab in pretreated patients with unresectable or metastatic cutaneous, uveal and mucosal melanoma. Med J Aust 2014;201:49-53.

67. Simeone E, Gentilcore G, Giannarelli D et al. Immunological and biological changes during ipilimumab treatment and their potential correlation with clinical response and survival in patients with advanced melanoma. Cancer Immunol Immunother 2014;63:675-83.

68. Weide B, Martens A, Hassel JC et al. Baseline biomarkers for outcome of melanoma patients treated with pembrolizumab. Clin Cancer Res 2016;22:5487-96.

69. Yuan J, Adamow M, Ginsberg BA et al. Integrated NYESO-1 antibody and CD8+ T-cell responses correlate with clinical benefit in advanced melanoma patients treated with ipilimumab. Proc Natl Acad Sci USA 2011;108:16723-8.

70. Martens A, Wistuba-Hamprecht K, Geukes Foppen M et al. Baseline peripheral blood biomarkers associated with clinical outcome of advanced melanoma patients treated with ipilimumab. Clin Cancer Res 2016;22:2908-18.

71. Vetizou M, Pitt JM, Daillere R et al. Anticancer immunotherapy by CTLA-4 blockade relies on the gut microbiota. Science 2015;350:1079-84. 